\title{
Influence of Triphenyllead Chloride on Biological and Model Membranes
}

Halina Kleszczyńska ${ }^{\mathrm{a}, *}$, Krzysztof Bielecki ${ }^{\mathrm{b}}$, Janusz Sarapuk ${ }^{\mathrm{a}}$, Anna Dziamska ${ }^{\mathrm{a}}$ and Stanisław Przestalski ${ }^{\mathrm{a}}$

a Department of Physics and Biophysics, Agricultural University, Norwida 25, 50-375 Wrocław, Poland. Fax: (+48)-71-32-05-172. E-mail: Halina@ozi.ar.wroc.pl

b Department of Botany and Plant Physiology, Cybulskiego 32, 50-205 Wrocław, Poland

* Author for correspondence and reprint requests

Z. Naturforsch. 55c, 764-769 (2000); received May 16/June 21, 2000

Triphenyllead Chloride, Toxicity, Lipid Membrane Destabilization

The physiological and hemolytic toxicities of triphenyllead chloride (TPhL) as well as its modyfying influence on model lipid membranes were studied. The experiments allowed the determination of $\mathrm{TPhL}$ concentrations causing $50 \%$ inhibition of growth of Spirodela oligorrhiza, Lemna minor and Salvinia natans $\left(\mathrm{EC}_{50}\right), 100 \%$ hemolysis of pig erythrocytes $\left(\mathrm{C}_{100}\right)$ and destabilization of planar lipid membranes (CC). Also, fluidity of erythrocyte ghosts was measured by fluorescence technique and osmotic sensitivity of erythrocytes to the presence of TPhL. All parameters studied were found to be dependent on $\mathrm{pH}$, of experimental solutions and the concentration of TPhL. Acidic conditions increased $\mathrm{EC}_{50}, \mathrm{C}_{100}$ and $\mathrm{CC}$ concentrations of TPhL. Fluorescence and osmotic measurements showed that osmotic stability and fluidity decreased with increasing trimethyllead concentration.

A possible mechanism of TPhL toxicity is discussed. It is assumed that $\mathrm{TPhL}$ is interacting with the lipid phase of the models used. It is also assumed that there may exist various, ionic and nonionic, forms of TPhL as a result of its speciation under different experimental conditions. These species, due to their differentiated lipophilicity, may exert different effects on the model membranes studied.

\section{Introduction}

Organolead compounds are anthropogenic toxic agents often found in the environment and mostly originating from antiknock additives to gasoline. However, some of them are introduced into the environment purposefully. Such is the case of triphenyllead compounds (TPhL) used as fungicides, bactericides, algicides, repellents or defoliants (Thayer, 1976; Eng et al., 1991; Kumari et al., 1993). They are also used as additives to antifouling paints that retain their toxicity over long periods (Thayer, 1976). Triphenyllead chloride was found to act as membrane toxicant causing hemolysis of red cells and modification of membrane channels (Trela et al., 1997). It effectively depolarized transmembrane potential of model membranes and caused intensive efflux of electrolytes from maize roots (Radecka et al., 1996). All these effects may be connected with direct or indirect interaction of $\mathrm{TPhL}$ with membrane lipids. It is known that toxicity of organometals depends on their hydrophobic chains and the polarity of the central metal ion (Ade et al., 1996). TPhL has a highly lipophilic character and is the most toxic compound of triorganolead compounds (Radecka et al., 1996). This lipophilicity and concurrent toxicity may change when the $\mathrm{pH}$ of the aqueous environment changes.

\section{Materials and Methods}

\section{Materials}

Triphenyllead (TPhL) was purchased from Alfa Products (Karlsruhe, Germany).

Planar lipid membranes (BLM) were formed from azolectin dissolved in a mixture of n-decane and n-butanol, all purchased from Sigma (St. Louis, USA). All used chemicals were of analytical grade.

Fluorescent probes DPH (1.6-diphenyl-1,3,5hexatriene) and TMA-DPH [(1-(4-trimethylammoniumphenyl)-6-phenyl-1,3,5-hexatriene-ptoluenesulfonate] were product of Molecular Probes Inc. (Eugene, USA).

\section{Erythrocyte experiments}

Fresh heparinized pig blood was used in the experiments, which were performed in phosphate 
buffers of different $\mathrm{pH}$ (5.0, 6.0, 7.4 and 8.0). Erythrocytes were washed four times in the phosphate buffer and incubated in it, after adding chosen concentrations of $\mathrm{TPhL}$, at $37^{\circ} \mathrm{C}$ for $4 \mathrm{~h}$. The hematocrit was $2 \%$. Percent of hemolysis was measured of $1 \mathrm{ml}$ samples taken after $0.5,1,1.5,2$, 3 and $4 \mathrm{~h}$ of incubation. They were centrifuged and the hemoglobin content of the supernatant was measured with a Specol 11 spectrophotometer (Carl Zeiss, Jena, Germany) at $540 \mathrm{~nm}$ wavelength. TPhL was dissolved in ethanol prior to adding to phosphate buffer (concentration of ethanol in the samples did not exceed $1 \%, v / v$ ).

Osmotic resistance was measured for erythrocytes modified for $0.5 \mathrm{~h}$ in solutions containing different $\mathrm{TPhL}$ concentrations $(1 \mu \mathrm{M}, 10 \mu \mathrm{M}, 20 \mu \mathrm{M}$ and $30 \mu \mathrm{M})$. Hematocrit was $2 \%$. The cells were then suspended in hypotonic solutions of $\mathrm{NaCl}$ and hemolysis was measured as described.

Fluorescence measurements were performed on erythrocyte ghosts labelled with DPH and TMADPH using the SFM spectrofluorimeter (KONTRON). The polarization coefficient was calculated according to the formula (Lakowicz, 1983; Campbell and Dwek, 1984; Lentz, 1988):

$$
P=\left(I_{\mathrm{II}}-G I_{\perp}\right) /\left(I_{\mathrm{II}}+G I_{\perp}\right),
$$

where $I_{\mathrm{II}}$ - intensity of fluorescence emitted parallel to the polarization plane of the exciting light, $I_{\perp}$ - intensity of fluorescence emitted perpendicular to the polarization plane, $G$ - diffraction constant, dependent on wavelength.

\section{Plant experiments}

Studies on the physiological toxicity of TPhL were done on axenically grown Spirodela oligorrhiza, Salvinia natans and Lemna minor. Two equal fronds of plants were placed in Erlenmayer flask containing modified Hoagland's solution (Knypl et al., 1976). Solutions of pH 6.2 were used in all cases. The plants were cultivated under constant illumination $120 \mu \mathrm{Em}^{-2} \mathrm{~s}^{-1}$ at $25^{\circ} \mathrm{C}$. After 8 days the dry mass of the plants was determined. Biomass data were expressed as percent control response. Calculation of the effective concentrations of $\mathrm{TPhL}$ resulting in $50 \%$ growth inhibition $\left(\mathrm{EC}_{50}\right)$ compared to controls were done by using non-linear regression (VanEwijk and Hoekstra, 1993).

\section{BLM experiments}

Planar lipid membranes were formed from $2.5 \%$ solution $(w / v)$ of azolectin in n-decane : n-butanol $(1: 1 \mathrm{v} / \mathrm{v})$ on a hole of $1.5 \mathrm{~mm}$ diameter in a Teflon two-chamber measuring cell. Concentrated triphenyllead chloride solution, with addition of methanol, was pipetted directly into the bath solution (phosphate buffer; pH 4.1, 6.1 and 8.0) until its concentration reached a value at which membrane life-time was not longer than $3 \mathrm{~min}$. This concentration is further referred to as the critical concentration (CC). The time necessary for a lipid membrane to achieve the bimolecular arrangement was about $15 \mathrm{~min}$ at room temperature $\left(\sim 22^{\circ} \mathrm{C}\right)$. This means that under the $\mathrm{CC}$ conditions no new membrane could be formed. The process of membrane formation was monitored optically. The measuring system consisted of a Keithley 617 Programmable Electrometer and a standard voltmeter controlling the DC voltage $(20 \mathrm{mV})$ applied to the membrane by means of calomel electrodes immersed directly into the buffer solution. It enabled continuous BLM monitoring. Each experiment was repeated at least three times.

\section{Results}

The results of the experiments with planar lipid membranes showed that TPhL efficacy to destroy the membranes depended on $\mathrm{pH}$ of the solution. No big differences in the $\mathrm{CC}$ values were found for the two highest values of $\mathrm{pH}\left(5.7 \times 10^{-5} \mathrm{M}\right.$ and $8.3 \times 10^{-5} \mathrm{M}$, for $\mathrm{pH} 6.1$ and 8.00 , respectively). A very significant decrease in $\mathrm{TPhL}$ efficiency to break bilayer lipid membranes was observed at $\mathrm{pH}$ 4.1. The critical concentration found for this buffer was about one order of magnitude higher compared with those for other $\mathrm{pH}$ values $\left(5.2 \times 10^{-4} \mathrm{M}\right)$.

Qualitatively similar results were obtained in the erythrocyte experiments where the weakest hemolysis of RBC was observed for $\mathrm{pH}$ 5.0. Hemolysis time-dependence curves for $\mathrm{pH} 7.4$ and 8.0 were practically the same (Fig. 1 ). They also show an increase of hemolysis with an increase of TPhL concentration. Increasing concentration of TPhL was also clearly influencing osmotic resistance of ghost erythrocytes as can be seen in Fig. 2 . The resistance decreased, which is evidenced by the right-handed gradual shift of the hemolytic 

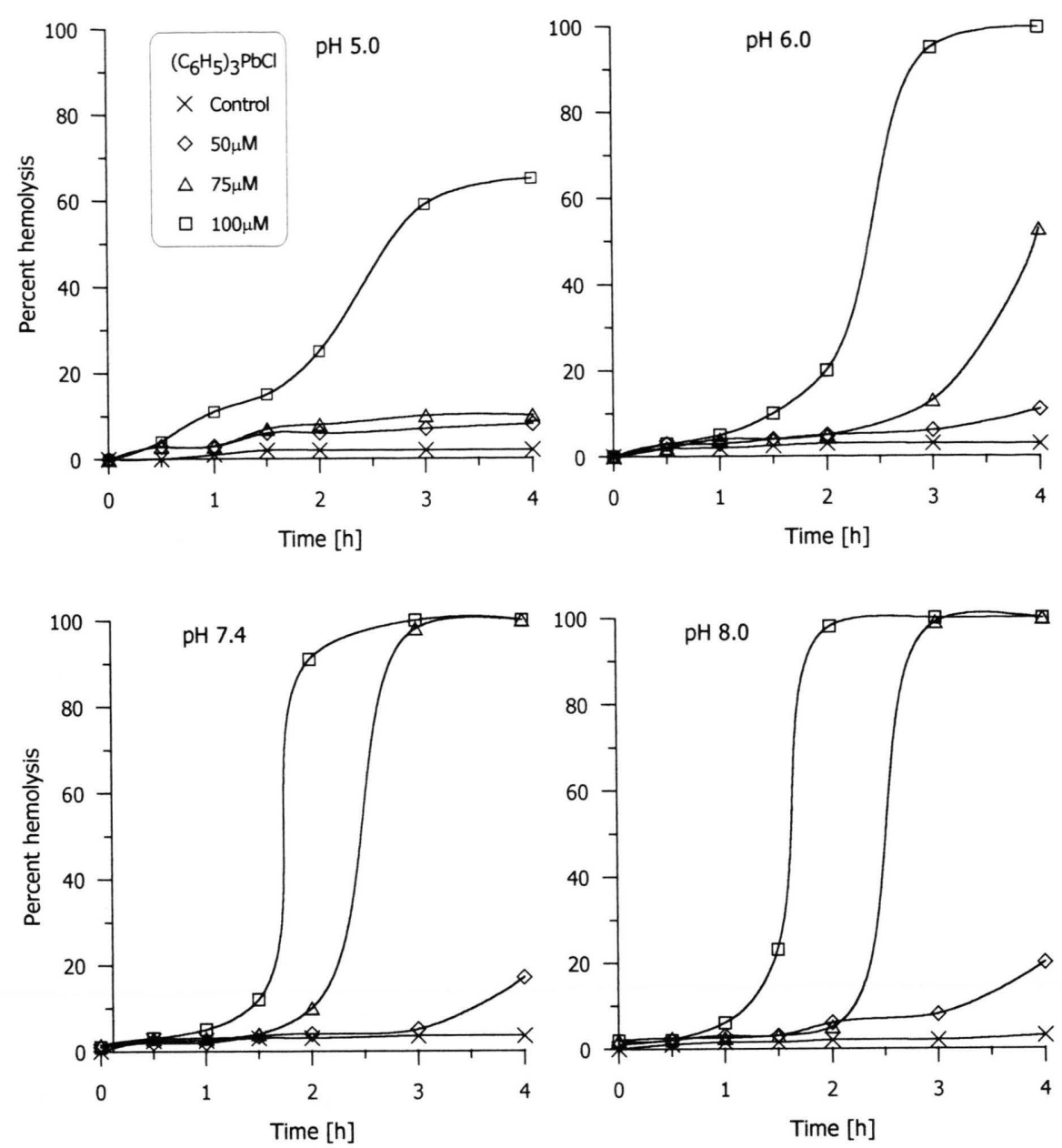

Fig. 1. The hemolysis time-dependence curves. Standard deviation did not exceed $6 \%$.

curves. TPhL concentration increase was also found to change fluidity of RBC membranes as seen in Fig. 3 where polarization coefficients calculated from fluorescence experiments are shown. An increase of this coefficient means a decrease of ghosts erythrocyte membranes fluidity.

Plant growth inhibition experiments are presented in Fig. 4. Two of the plants studied, Spirodela oligorrhiza and Lemna minor exhibited similar sensitivity towards TPhL. Calculated inhibition coefficients $\left(\mathrm{EC}_{50}\right)$ were $17.7 \mathrm{~nm}$ (standard deviation was $1.3 \mathrm{~nm}$ ) and $29 \mathrm{~nm}$ (standard deviation was $3.0 \mathrm{~nm}$ ) for Spirodela oligorrhiza and Lemna minor, respectively. Salvinia natans was much more resistant to $\mathrm{TPhL}$ and its $\mathrm{EC}_{50}$ was $815 \mathrm{~nm}$ (standard deviation was $34 \mathrm{~nm}$ ).

\section{Discussion}

As expected, the levels of concentrations of triphenyllead chloride causing the observed changes in biological and model objects studied were different. However, there are some common features of TPhL action on those objects. The increase of concentration of $\mathrm{TPhL}$ makes its toxic effects more evident (Figs 1, 2 and 4). Hemolytical toxicity was found to be $\mathrm{pH}$ dependent (Fig. 1) and corresponding to BLM destabilization efficiency. The obvious conclusion seems to be that TPhL interaction with lipids of RBC or BLM membrane must be in a way connected with changes concerning both TPhL and the objects studied and which follow changes of the experimental conditions; in this 


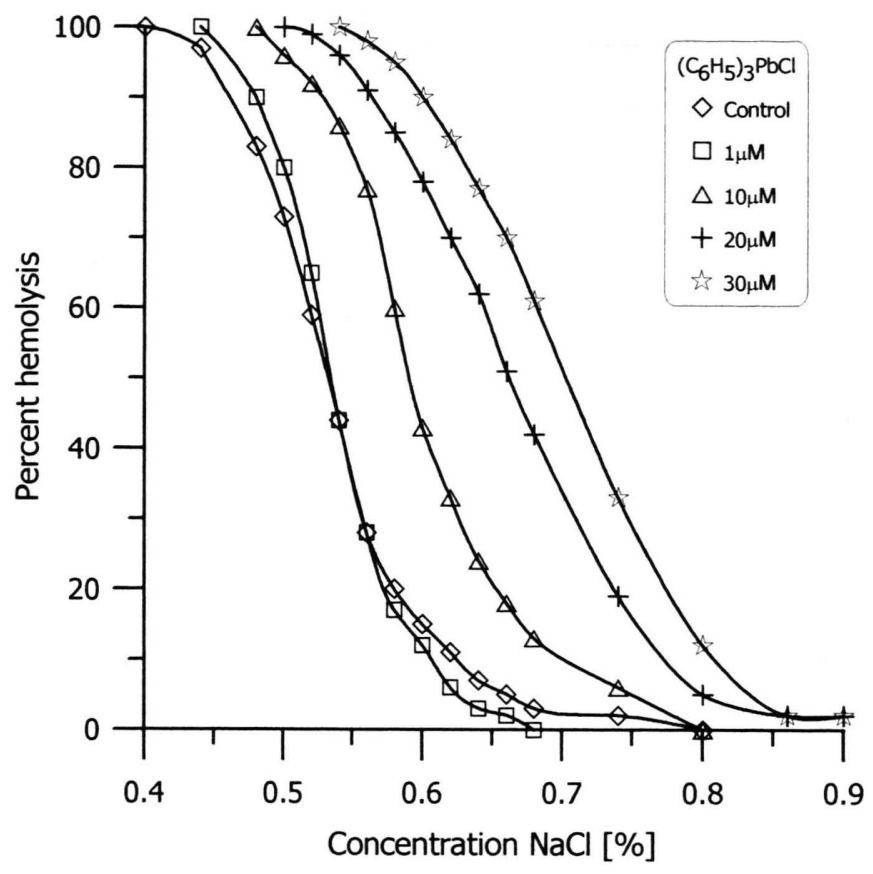

Fig. 2. The osmotic resistance of erythrocytes in the presence of triphenyllead chloride (TPhL). Standard deviation did not exceed $6 \%$. case $\mathrm{pH}$ changes. There are at least two possibilities. Maybe the more acidic environment changes the surface potential of the lipid bilayer weakening the electric interaction between the bilayer, especially with phosphate group of lipids, and TPhL. Since this interaction is known to contribute in the overall interaction of amphiphilic compounds, including organometallic ones, with biological and model membranes (Kleszczyńska et al., 1990; Gabrielska et al., 1997) its change can lead, to some degree, to the observed effects. Another possibility is a speciation of TPhL, under different $\mathrm{pH}$ conditions, into various forms. In water solution TPhL may dissociate as follows (Komiya and Kohi, 1976):

$$
\mathrm{Ph}_{3} \mathrm{PbCl}+\mathrm{n} \cdot \mathrm{H}_{2} \mathrm{O} \leftrightarrow \mathrm{Ph}_{3} \mathrm{~Pb}^{+} \mathrm{n} \cdot \mathrm{H}_{2} \mathrm{O}+\mathrm{Cl}^{-} .
$$

Equilibrium of this reaction may shift depending on $\mathrm{pH}$ of the solution and the overall interaction depends on the ratio of the dissociated to undissociated molecules. A shift towards the non-ionic form means there are more molecules of the lipophilic character. It is also possible that there appear other species, e. g., $\mathrm{Ph}_{3} \mathrm{~Pb}(\mathrm{OH}) \mathrm{PbPh}_{3}+n \cdot \mathrm{H}_{2} \mathrm{O}$, as it is observed in the case of trialkylleads (Tobias, 1978). Interaction of those species with the ob- jects studied may be different due to their different lipophilicity which determines the possibility of particular species entering into the lipid bilayer. As a result, different efficiencies of $\mathrm{TPhL}$ in solutions at different $\mathrm{pH}$ are observed.

The results of the plant experiments do not point directly to the lipid phase of biological membranes as the place where the toxic action of $\mathrm{TPhL}$ is localized. The calculated values of $\mathrm{EC}_{50}$, especially those obtained for Lemna minor and Spirodela oligorrhiza, are much lower than the concentrations causing RBC hemolysis or BLM destruction. However, it must be remembered that the concentration of biologically active agent necessary to cause a change in the mechanical properties of membrane are usually high. Only studies of some more sensitive parameters may answer if the lipid phase of the membrane is the target of the action of such agents. In this case we used the fluorescence technique to detect any changes in erythrocyte ghost membranes caused by subhemolytic concentrations of TPhL. Indeed, it was found that very small concentrations of $\mathrm{TPhL}$ changed fluidity of those membranes. The increase of membrane rigidity with increase of $\mathrm{TPhL}$ concentration, roughly linear, is the evidence that $\mathrm{TPhL}$ 


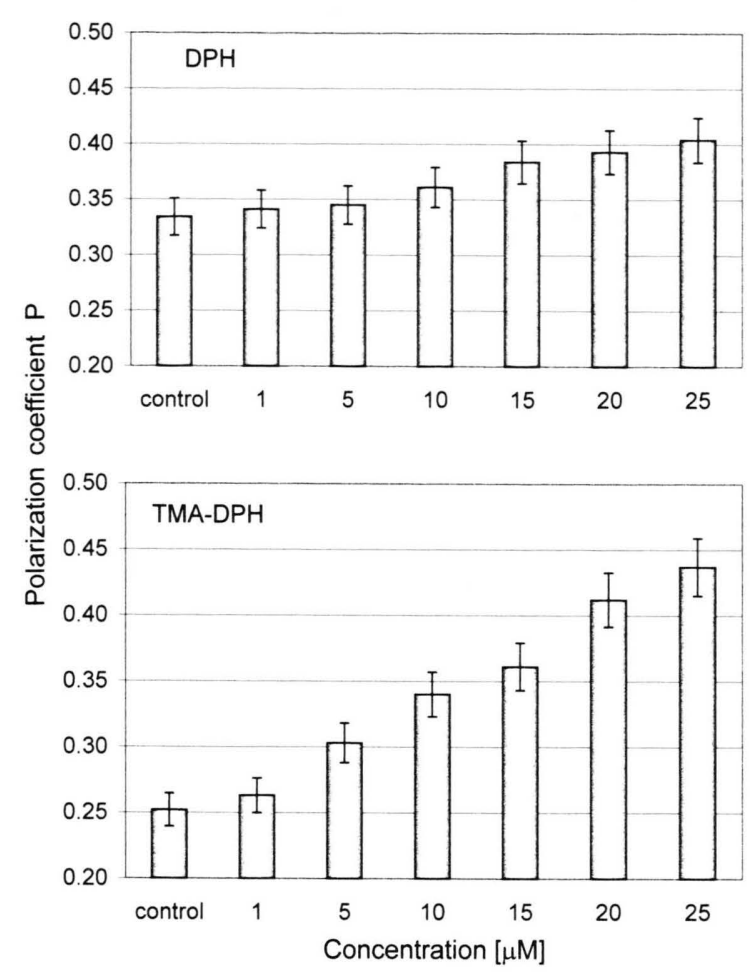

Fig. 3. The change of the polarization coefficient induced by the increase of the concentration of triphenyllead chloride (TPhL). DPH (1,6-diphenyl-1,3,5-hexatriene) and TMA-DPH [(1-(4-trimethylammoniumphenyl)-6phenyl-1,3,5-hexatriene p-toluenesulfonate] - fluorescent probes used. molecules, and/or their species, incorporate into the lipid phase (Fig. 3). Similar conclusion follows from studies on osmotic resistance of erythrocytes.

On the basis of the experiments performed it can be assumed that there is evidence that the biological activity of TPhL may be connected with its interaction with the lipid phase of biological membranes, even if its concentration is not causing "observable" external damages. The known examples of triorganoleads influence on metabolic processes in biological objects (Hager et al., 1987; 1989; Endres and Faulstich, 1989) must not be necesserily connected with their direct action on the protein phase of those objects. It is possible that the modifications of the lipid phase of biological membranes induce further biological effects via the lipid-protein interactions.

\section{Acknowledgements}

This work was sponsored by the Polish Research Committee (KBN, grant no. 6PO4 GO 4313.

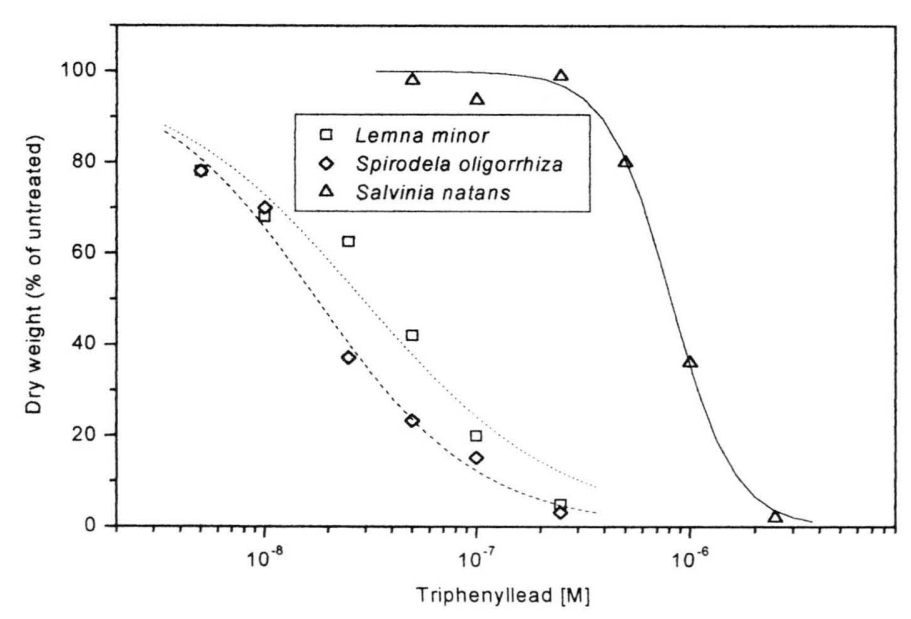

Fig. 4. The inhibition of plant growth by triphenyllead chloride (TPhL). Calculated inhibition coefficients $\left(\mathrm{EC}_{50}\right)$ and standard deviations are given in Results. 
Ade T., Zaucke F. and Krug H. F. (1996), The structure of organometals determines cytotoxicity and alteration of calcium homeostasis in HL-60 cells. Fresenius' J. Analyt. Chem., Abs. 354, 609-614.

Campbell L. D. and Dwek R. A. (1984), Biological Spectroscopy. Benjamin Cummings Publ. Menlo Park and London, pp. 91-120.

Endres K. P. and Faulstich H. (1989), Triethyllead inhibits mitochondrial ATPase of the endosperm and sucrose uptake in the cotyledons of Ricinus communis. J. Plant Physiol. 135, 531-536.

Eng G., Tierney E. J., Olson G. J., Brinckman F. E. and Bellama J. M. (1991), Total surface areas of group IVA organometallic compounds: predictors of toxicity to algae and bacteria. Appl. Organomet. Chem. 5, 33-37.

Gabrielska J., Sarapuk J. and Przestalski S. (1997), Role of hydrophobic and hydrophilic interactions of organotin and organolead compounds with model lipid membranes. Z. Naturforsch. 52c, 209-216.

Hager A., Moser I. and Berthold W. (1987), Organolead toxicity in plants: triethyl lead $\left(\mathrm{Et}_{3} \mathrm{~Pb}^{+}\right)$acts as a powerful transmembrane $\mathrm{Cl}^{-} / \mathrm{OH}^{-}$exchanger dissipating $\mathrm{H}^{+}$-gradients at nano-molar levels. Z. Naturforsch. 42c, $1116-1120$.

Hager A., Stransky H. and Berthold W. (1989), Uncoupling of photophosphorylation by triethyllead $\left(\mathrm{Et}_{3} \mathrm{~Pb}^{+}\right)$generated from tetraethyllead $\left(\mathrm{Et}_{4} \mathrm{~Pb}\right)$ in illuminated chloroplasts. J. Plant Physiol. 134, 5-8.

Kleszczyńska H., Sarapuk J., Przestalski S. and Kilian M. (1990), Mechanical properties of red cell and BLM in the presence of some mono- and bis-quaternary ammonium salts. Stud. Biophys. 135, 191-199.

Knypl J. S., Witek S. and Oświẹcimska M. (1976), Growth retarding effect of N,N-dimethylmorpholinium chloride and CCC in Spirodela oligorrhiza. Z. Pflanzenphysiol. 79, 53-61.
Komiya S. and Kohi J. (1976), The conductometric behaviour of organometallic chlorides from IVB. J. Am. Chem. Soc. 98, 7599-7609.

Kumari A., Tandon J. P. and Singh R. V. (1993), Antimicrobial effects of newly synthesized organotin(IV) and organolead(IV) derivatives. Appl. Organomet. Chem. 7, 655-660.

Lakowicz J. R. (1983), Principles of Fluorescence Spectroscopy. Plenum Press, New York and London, pp. $112-151$.

Lentz B. R. (1988), Membrane "fluidity" from fluorescence anisotropy measurements. In: Spectroscopic Membrane Probes. (L. M. Loew ed.). CRC Press, Boca Raton, FL., Vol. 1, 13-41.

Radecka H., Sarapuk J., Zielińska D., Przestalski S., Radecki J. and Cieciórski B. (1996), An attempt to explain the toxic properties of alkylolead derivatives towards plants on the base of studies on model lipid membranes. J. Med. Prev. 4, 87-92.

Thayer J. S. (1974), Organometallic compounds and living organisms. J. Organomet. Chem. 76, 265-295.

Tobias R. S. (1978), The chemistry of organometallic cations in aqueous media. In: Organometals and Organometalloids (F. E. Brinckman and J. M. Bellama, eds.). Am. Chem. Soc., pp. 130-147.

Trela Z., Radecki J. and Przestalski S. (1997), Effect of triphenyllead chloride on the resting potential and electrical conductance of Nitellopsis obtusa membrane. Polish J. Environ. Stud. 6, 37-40.

VanEwijk P. H. and Hoekstra A. (1993), Calculation of the $\mathrm{EC}_{50}$ and its confidence interval when subtoxic stimulus is present. Ecotoxicol. Environ. Saf. 52, 25-32. 